Revue d'histoire de l'Amérique française

REVUE D.HISTOIRE DE L'AMÉRIQUE FRANÇAISE

\title{
HAEFELI, Evan et Kevin SWEENEY, Captors and Captives. The 1704 French and Indian Raid on Deerfield (Boston, University of Massachusetts Press, 2003), 376 p.
}

\section{Maxime Gohier}

Volume 59, numéro 1-2, été-automne 2005

URI : https://id.erudit.org/iderudit/012735ar

DOI : https://doi.org/10.7202/012735ar

Aller au sommaire du numéro

Éditeur(s)

Institut d'histoire de l'Amérique française

ISSN

0035-2357 (imprimé)

1492-1383 (numérique)

Découvrir la revue

Citer ce compte rendu

Gohier, M. (2005). Compte rendu de [HAEFELI, Evan et Kevin SWEENEY, Captors and Captives. The 1704 French and Indian Raid on Deerfield (Boston, University of Massachusetts Press, 2003), 376 p.] Revue d'histoire de l'Amérique française, 59(1-2), 150-153. https://doi.org/10.7202/012735ar d'utilisation que vous pouvez consulter en ligne.

https://apropos.erudit.org/fr/usagers/politique-dutilisation/ 
chapitre 5 dont les données sur les travailleurs et les relations de travail sont traitées à la lumière de deux études portant sur la base de Portsmouth en Angleterre. De plus, l'auteur situe le chantier de carénage sur la carte de l'Empire mais pas dans son contexte canadien. On sait ainsi qu'Halifax est l'enfant pauvre des chantiers navals, suivant loin derrière, quant aux dépenses consenties par la métropole, les Bermudes, Madras, Malte et Trincomalee (malgré les 1,55 millions $£$ que coûte le chantier durant la période sous enquête, p. 41). Par contre, il ignore presque totalement Québec et même Kingston qui, pendant la guerre de 1812, abritent un chantier de construction naval, un statut que n'atteindra jamais Halifax. La tâche de tisser des liens entre le sujet traité ici et les colonies sœurs de l'Amérique reviendra à un(e) autre historien(ne).

Ces dernières réserves mises à part, il faut saluer ce superbe effort de compréhension d'un sujet complexe. Au total, Ashore and Afloat allie brillamment une démarche d'histoire navale avec de nombreuses autres: militaire, administrative, économique et sociale, comme peu de livres ont réussi à le faire.

ROCH LEGAULT

Département d'histoire Collège militaire royal du Canada

HAEFELI, Evan et Kevin SWEENEY, Captors and Captives. The 1704 French and Indian Raid on Deerfield (Boston, University of Massachusetts Press, 2003), 376 p.

Trois cents ans après ce qui fut l'un des événements militaires les plus importants de l'histoire de la Nouvelle-France, l'ouvrage de Evan Haefeli et Kevin Sweeney jette un regard tout à fait nouveau sur l'attaque francoamérindienne contre le village de Deerfield, au Massachusett. Comme son titre le laisse entendre, Captors and Captives se veut d'abord et avant tout une étude des individus ayant participé à l'événement. Son originalité repose d'ailleurs sur une recherche colossale ayant permis de retracer les antécédents de même que le destin des différents protagonistes, tant captifs anglais que ravisseurs français et amérindiens.

Cette analyse biographique est d'autant plus intéressante qu'elle s'inscrit résolument dans la mouvance des nouvelles études sur la "frontière», qui s'intéressent principalement aux contacts entre individus d'origines culturelles distinctes. Elle permet en effet aux auteurs d'adopter une multitude de perspectives et de prendre en compte les intérêts et les moti- 
vations des différents acteurs. Elle permet aussi de mettre en lumière les relations de conflits et d'accommodements nées de la rencontre entre ces individus. Par cette démarche, les auteurs visent à nuancer les stéréotypes et les généralisations héritées de l'historiographie américaine des $\mathrm{XIX}^{\mathrm{e}}$ et $\mathrm{xx}^{\mathrm{e}}$ siècles, qui présentait souvent l'événement comme le résultat d'un clash culturel et impérial.

L'ouvrage se divise en quatre sections. Il s'ouvre d'abord sur une présentation des trois "communautés» impliquées dans l'événement (Français, Anglais et Amérindiens) et des causes de l'attaque. L'accent est mis avant tout sur les intérêts distincts qu'avaient les Français et leurs alliés à prendre les armes et à s'engager dans le conflit impérial que fut la guerre de Succession d'Espagne. La seconde section s'intéresse au raid lui-même. Quoique l'analyse soit plus événementielle, ce sont encore ici les relations interpersonnelles qui priment: les Français et les Amérindiens qui visitèrent Deerfield dans les mois précédant le raid; la coordination des troupes lors de l'assaut; les relations entretenues par les ravisseurs amérindiens avec leurs captifs durant la marche de trois semaines qui devait les conduire à Montréal. En troisième partie, l'ouvrage analyse le sort des 89 captifs qui arrivèrent vivants en Nouvelle-France et notamment les forces multiples qui contribuèrent à les intégrer dans leur communauté d'accueil ou à faciliter leur retour en Nouvelle-Angleterre. Enfin, une quatrième section s'attarde aux transformations subies par les communautés à la suite du raid, de même qu'à l'influence de la captivité sur les individus.

L'approche individuelle adoptée par les auteurs fait clairement ressortir le caractère ambigu des acteurs historiques, dont le comportement est à la fois conditionné par les forces structurelles qui les entourent et par leur volonté de forger leur propre destin (p. 4 et 7). Discutant des motivations françaises et amérindiennes pour lancer un raid contre Deerfield, les auteurs insistent tout autant sur les intérêts individuels de chacun que sur le poids des politiques impériales. Ils mettent alors en lumière un important contraste entre, d'une part, «l'enthousiasme» des officiers français à rassembler et conduire les troupes afin de promouvoir leur ascension sociale (chapitre II) et, d'autre part, des chefs Abénaquis dont les efforts pour demeurer neutres dans le conflit furent contrecarrés par les manœuvres politiques françaises et les attaques anglaises (chapitre IV). Ainsi, les empires apparaissent moins comme des blocs figés que comme des « toiles diaphanes ", influençant la vie des individus tout autant qu'ils dépendent de leurs actions pour se déployer (p. 5). 
Sous la plume de Haefeli et Sweeney, les frontières culturelles sont elles aussi marquées par une certaine dichotomie, se présentant comme des limites aussi bien fixes que poreuses. La religion apparaît notamment comme la principale barrière à l'intégration des captifs anglais dans leurs communautés d'accueil. Par ailleurs, si Français et Amérindiens entretenaient une relation d'alliance, les différences culturelles rendaient celle-ci très fragile, "full of tension and mistrust» (p. 145). Des frontières existaient donc bel et bien entre les groupes, qui les préservaient parfois délibérément. Pourtant, rien n'empêchait un individu de les traverser, voire de les retraverser à sa guise: «Even during wartime, affirment les auteurs, the frontier did not present an impenetrable barrier to individuals who wanted to cross it» (p. 95). Les auteurs rejettent donc le concept de middle ground, popularisé par l'historien Richard White, qui insiste sur l'adaptation à la culture de l'Autre dans les situations de contact. Car selon eux, si adaptation il y eut dans le contexte du raid contre Deerfield, «it was fondumentally about relations between individuals» et «it usually did not last long» (p. 4). Deerfield, par exemple, émergea à la suite du raid comme un point de convergence des relations anglo-amérindiennes, et ce, parce que les ex-captifs s'impliquèrent davantage dans la guerre contre les Abénaquis, mais aussi parce que le village devint un centre diplomatique important, alors que des liens encourageaient les ravisseurs amérindiens à visiter leurs anciens captifs.

L'image qui émane d'une telle étude est évidemment multiple et fragmentée : les tendances y côtoient les cas d'exceptions et les conflits alternent avec les situations d'accommodement. En bout de ligne, ce qui semble primer, c'est la diversité des expériences vécues, comme en témoigne l'analyse que font les auteurs de la captivité. Ils révèlent en effet que le cas de la célèbre Eunice Williams (la fille du pasteur John Williams, qui passa le reste de ses jours chez les Iroquois de Kanawake) est relativement atypique, car la plupart des captifs ayant choisi de demeurer en Nouvelle-France s'établirent non pas chez les Amérindiens, mais dans la société canadienne, au sein de laquelle ils avaient tissé des liens économiques et sociaux. Cette diversité est en partie le résultat de la situation particulière dans laquelle s'est retrouvé chaque captif: «Depending on the community - even the family — in which captives found themselves, they could be used as slaves or servants, adopted as family members, bartered for money and ransomed, retained as hostages, or even tortured to death.» Mais les choix effectués par chacun pouvaient aussi être lourds de conséquences sur son destin: «Economic calculation, cultural pride, 
family ties, and human frailty were important too in determining the fate of individuals. » (p. 145)

En somme, Captors and Captive est un ouvrage fort intéressant, qui évacue toute explication réductrice et déborde largement le cadre restreint de l'événement auquel il s'intéresse pour s'attaquer à des questions fondamentales de l'histoire coloniale, telles que l'intégration des communautés nord-américaines aux empires européens de même que le rôle des frontières dans les sociétés nouvelles.

MAXIME GOHIER

Département d'histoire

Université du Québec à Montréal

HARRIS, Richard, Creeping Conformity: How Canada Became Suburban, 1900-1960 (Toronto, University of Toronto Press, coll. «Themes in Canadian History», 2004), 205 p.

Depuis quand le Canada est-il devenu une nation suburbaine, suivant de ce fait une trajectoire tracée par des pays comme l'Australie et les ÉtatsUnis? Quelles forces politiques et économiques ont façonné les espaces qui se trouvent en périphérie des villes-centres? Qu'entend-on par banlieue et quels modes de vie y sont associés? Quels groupes sociaux y habitent? Divisé en sept chapitres, incluant l'introduction et la conclusion, l'ouvrage de synthèse de Richard Harris répond à ces questions en nous exposant une histoire où sont engagées des dynamiques économiques et sociopolitiques ayant soutenu le développement des espaces suburbains des régions métropolitaines canadiennes entre les années 1900 et 1960. Parmi les facteurs à l'œuvre, Harris fait ressortir les mouvements migratoires, la déconcentration de l'emploi, le redéploiement de la mobilité, l'action des pouvoirs publics locaux et supérieurs, de même que les aspirations des ménages à devenir propriétaires afin d'améliorer leurs conditions de vie et de logement.

La principale thèse mise de l'avant par l'auteur est que la banlieue, telle que mise en forme au début $\mathrm{du} \mathrm{xx}^{\mathrm{e}}$ siècle, a progressivement perdu de sa diversité sociale et fonctionnelle initiale pour devenir, entre les années 1945 et 1960, un milieu homogénéisant et conformiste. Pour étayer sa thèse, Harris utilise une approche sociospatiale présentée dans le deuxième chapitre. Le troisième chapitre vise à éclairer les interrelations qui prévalent entre l'urbanisation et les mutations de la périphérie, les banlieues s'inscrivant en continuité avec le processus d'urbanisation. 